\title{
Extract of Paecilomyces hepiali mycelia induces lipolysis through PKA-mediated phosphorylation of hormone-sensitive lipase and ERK-mediated downregulation of perilipin in 3T3-L1 adipocytes
}

Mei Ge, Rui Guo, Hai-xia Lou and Wen Zhang ${ }^{*}$

\begin{abstract}
Background: Cordyceps sinensis has been used for centuries in China as one of the most valued herbal medicine and tonic food. Paecilomyces hepiali, a fungal strain isolated from natural C. sinensis, has been used widely as a substitute of $C$. sinensis in medicine and health food. P. hepiali has been reported to have various pharmaceutical benefits, including triglyceride-lowing activity. However, its effects on triglyceride metabolism in adipocytes remain unknown. The purpose of the present study was to evaluate the effect of $P$. hepiali mycelia on adipocyte lipolysis and to clarify the underlying mechanisms.

Methods: The fully differentiated 3T3-L1 adipocytes were treated with methanol extract of Paecilomyces hepiali mycelia (PHME). Contents of glycerol released into the culture medium and intracellular triglyceride were measured as indices of lipolysis using glycerol assay kit and Oil red O staining, respectively. Then, effects of PHME on the main lipases or kinases involved in lipolysis regulation were investigated. Protein expression of adipose triglyceride lipase (ATGL) and perilipin, as well as phosphorylation of hormone-sensitive lipase (HSL), AMP-activated protein kinase (AMPK), and mitogen-activated protein kinases (MAPKs) were determined by western blotting. Moreover, nucleosides, important constituents of PHME, were analyzed using high performance liquid chromatography (HPLC).

Results: Treatment with PHME led to a significant increase in glycerol release thereby reduced intracellular triglyceride accumulation in fully differentiated adipocytes. PHME upregulated protein kinase (PK) A-mediated phosphorylation of HSL at serine residues of 563 and 660. Meanwhile, PHME treatment also upregulated phosphorylation of extracellular signalregulated kinase (ERK), and downregulated the protein level of perilipin. Pretreatment with the PKA inhibitor, H89, blunted the PHME-induced lipolysis and the phosphorylation of HSL (Ser 563 and 660). Moreover, pretreatment with ERK inhibitor, PD98059, weakened the PHME-caused glycerol release and downregulation of perilipin expression. HPLC analysis indicated there were adenosine, cordycepin, uridine and vernine in PHME.
\end{abstract}

Conclusions: Our results showed that PHME significantly induced lipolysis in 3T3-L1 adipocytes, which is mainly mediated by activation of HSL through PKA pathway and by downregulation of perilipin through activation of ERK pathway.

Keywords: Paecilomyces hepiali, 3T3-L1 adipocyte, Lipolytic effect, PKA, ERK

* Correspondence: wzhang@bio.ecnu.edu.cn

School of Life Sciences, East China Normal University, 500 Dongchuan Road,

Shanghai 200241, China

(c) The Author(s). 2018 Open Access This article is distributed under the terms of the Creative Commons Attribution 4.0 International License (http://creativecommons.org/licenses/by/4.0/), which permits unrestricted use, distribution, and reproduction in any medium, provided you give appropriate credit to the original author(s) and the source, provide a link to the Creative Commons license, and indicate if changes were made. The Creative Commons Public Domain Dedication waiver (http://creativecommons.org/publicdomain/zero/1.0/) applies to the data made available in this article, unless otherwise stated. 


\section{Background}

Cordyceps sinensis has been used for centuries in China as one of the most valued traditional Chinese medicine and tonic food $[1,2]$. In China, it is found in the soil of prairies at elevations of 3500-5000 m, mainly in the provinces of Qinghai, Tibet, Sichuan, Yunnan, and Gansu [3]. It has a wide range of pharmaceutical benefits, such as treatment for kidney and lung disorders, hemostatic and expectorant effects, anti-cancer, immune modulation, hypolipidemic and hypoglycemic activities [4-7]. Because of the limited habitat and over exploitation by humans, $C$. sinensis has been scarce in the nature. Therefore, its usage is limited due to the limited natural resource and high price [8]. Since 1970s, many scientists have been making much efforts to find the alternative material based on fermentation and cultivation of fungal species isolated from natural $C$. sinensis [8]. Among the colonized fungi in $C$. sinensis, Paecilomyces hepiali is one of the most important species [9]. The mycelia of this fungus are cultivated by artificial deep fermentation, and have been used widely as a substitute of $C$. sinensis in medicine and health foods, such as 'JinShuiBao' capsule, the commercial product of $P$. hepiali mycelia, has been used in clinics throughout China [3]. P. hepiali has been reported to exhibit anti-oxidation [10], anti-fatigue [11], anti-nociceptive [12], anti-tumor [13], anti-inflammatory [14], anti-diabetic [15] and antihyperlipidic effects $[3,15]$. The previous works of our Lab found that methanol extract from mycelia of $P$. hepiali (PHME) reduced fat accumulation in adipocyte via suppressing preadipocyte differentiation (Data not shown). These findings suggested this fungus has potential on regulating the metabolism of triglyceride.

It is known that triglyceride (TG) is the major energy storage form in mammals. The major site for storage of TG is the white adipose tissue [16]. In addition as a passive site for storage of energy in the form of TG, adipose tissue is considered as an important endocrine gland which secretes several bioactive molecules $[17,18]$. White adipose tissue stores TG during periods of energy excess, and hydrolyzes TG (lipolysis) to release fatty acids (FA) for use by other tissues during times of energy need [19]. However, a dysregulation of lipolysis may lead to metabolic abnormalities. Reduced lipolytic activity may contribute to accumulation of TG in adipose tissue and thus led to obesity, a major risk factor for metabolic disorders including type 2 diabetes and cardiovascular disease [19, 20]. Thus, strategies aimed at increasing lipolysis might be useful in preventing obesity and metabolic diseases [19].

Unlike TG synthesis that occurs in other organs, lipolysis is unique to adipocytes [19]. Adipose lipases perform significant functions in lipolysis because they catalyze the steps of hydrolysis and cleave TGs, diglycerides, and monoglycerides at various stages [21]. Adipose triglyceride lipase (ATGL) and hormone sensitive lipase (HSL) are quantitatively the most important lipases [22]. Activity of HSL is regulated by PKA-catalyzed phosphorylation at serine residues of 563, 659 and 660 [23, 24]. Other kinases, including AMPK, ERK, glycogen synthase kinase-4, and $\mathrm{Ca}^{2+} /$ calmodulin-dependent kinase, also phosphorylate HSL to modulate its enzyme activity [24]. Besides ATGL and HSL, perilipin A, a lipid droplet-associated protein, has also been considered as the major regulator for lipolysis in adipocytes [25]. Upon hormonal stimulation, perilipin A undergoes phosphorylation by PKA, and then facilitates the translocation of HSL to the lipid droplet and catalyses the lipolysis. However, perilipin A limits lipase access to the lipid droplet, thereby suppressing lipolysis in basal conditions [22, 24]. Therefore, decreased protein expression may impair the barrier function of perilipin A and subsequently lead to the increase in lipolysis. During this process, ERK activation is an early signal for the reduction in perilipin protein expression and subsequent induction of lipolysis [26].

Methanol extract from mycelia of Paecilomyces hepiali (PHME) have been shown to decrease TG level in serum and suppress adipogenesis in adipocytes. However, effect of PHME on lipolysis is relatively limited. Whether PHME can modulate HSL or perilipin A to affect lipolysis is still unknown. Therefore, the aim of this study was to clarify the effect of PHME on lipolysis, HSL and perilipin A, and further to elucidate the underlying mechanism in adipocytes.

\section{Methods}

\section{Chemicals and reagents}

Dulbecco's Modified Eagle Medium (DMEM) was purchased from Life Technologies (Carlsbad, CA). Fetal bovine serum and newborn calf serum were supplied by Bovogen Biological (Melbourne, Australia). Penicillin and streptomycin were purchased from Gibco (New York, NY). 3-isobutyl-1-methylxanthine (IBMX) and indomethacin (Indo) were from Aladdin Inc. (Shanghai, China). Trypsase was purchased from Shanghai Peiyuan biotechnology Co. Ltd. Dexamethasone (DEX), Oil Red O and MTT were supplied by Sigma (St Louis, MO). Bovine serum albumin (BSA) was from MP (Santa Ana, CA).

\section{Preparation of extract}

Dried mycelia of $P$. hepiali were kindly provided by Prof. Yu-quan Xu (Shanghai Jiaotong University, Shanghai, China), and the voucher specimen (No.: Xu Y.Q. 2,015,002) was deposited in HSNU. The $P$. hepiali strains were originally isolated, identified and fermented by Prof. Xu's laboratory. According to the previous report, methanol extract of $P$. hepiali mycelia was prepared with some modifications [27]. Dried mycelia were crushed and then extracted with 20 volumes of $100 \%$ methanol three times ( $3 \mathrm{~h}$ each) at $70{ }^{\circ} \mathrm{C}$ under reflux. After filtration, the methanol-extracted solution was concentrated and further dried in vacuum drying oven to produce the methanol extract of 
P. hepiali mycelia (PHME). The PHME was stored in a dark dryer at room temperature.

\section{Cell culture and differentiation}

3T3-L1 mouse embryonic fibroblasts were obtained from the National Center for Drug Screening (Shanghai, China). Preadipocyte differentiation was induced as described previously [28]. Briefly, 3T3-L1 preadipocytes were cultured in high-glucose DMEM supplemented with 10\% newborn calf serum and antibiotics $(100 \mu \mathrm{g} / \mathrm{mL}$ streptomycin and $100 \mathrm{U} / \mathrm{mL}$ penicillin) at $37^{\circ} \mathrm{C}$ under a $5 \% \mathrm{CO}_{2}$ atmosphere. After confluence, 3T3-L1 preadipocytes were maintained in culture medium for two days to induce differentiation (Day 0). Then, cells were incubated in differentiation medium 1 (DM1: DMEM containing $10 \mu \mathrm{g} / \mathrm{mL}$ insulin, $0.5 \mu \mathrm{M}$ IBMX, $1 \mu \mathrm{M}$ DEX, $0.2 \mathrm{mM}$ indomethacin, and $10 \%$ fetal bovine serum) for three days (Day 3). Then, the medium was changed to differentiation medium 2 (DM2: DMEM containing $10 \mu \mathrm{g} / \mathrm{mL}$ insulin and 10\% fetal bovine serum) for additional three days (Day 6). Subsequently, the cells were maintained in DMEM containing $10 \%$ fetal bovine serum for another two days (Day 8). Afterward, the fully differentiated adipocytes were harvested for further experiments.

\section{Cell viability assay}

MTT assay was used to evaluate the effect of PHME on cell viability of 3T3-L1 adipocytes [29]. Briefly, 3T3-L1 preadipocytes were seeded in 96 well plates, after differentiation to mature adipocytes (8 days), adipocytes were incubated in DMEM containing $0.2 \%$ BSA for $12 \mathrm{~h}$. Afterward, replaced the media with DMEM containing 0.2\% BSA and various concentrations of PHME for $48 \mathrm{~h}$. After incubation, $20 \mu \mathrm{L}$ of $3 \mathrm{mg} / \mathrm{mL}$ MTT was added in each well and incubated for $4 \mathrm{~h}$. Then, the culture medium was removed and added $200 \mu \mathrm{L}$ DMSO in each well. The plate was placed for $20 \mathrm{~min}$ at room temperature and then the absorbance was detected at $490 \mathrm{~nm}$ on a plate reader (BioTek Inc., VT, USA).

$$
\text { Cell viability } \%=\frac{\text { ODsample }- \text { ODblank }}{\text { ODcontrol }- \text { ODblank }} \times 100 \%
$$

\section{Oil red $O$ staining}

To measure the intercellular lipid accumulation in fully differentiated adipocytes, cells were stained with oil red $\mathrm{O}$ as described by Wang [28]. Adipocytes were washed twice with phosphate-buffered saline (PBS, pH 7.4) and fixed with $10 \%$ formalin in PBS at room temperature for at least $1 \mathrm{~h}$ and then washed with $60 \%$ isopropanol. After that, $500 \mu \mathrm{L}$ of $0.21 \%(w / v)$ oil red $\mathrm{O}$ solution (working solution) was added in each well to stain the lipids for $10 \mathrm{~min}$ at room temperature. Freshly diluted oil red O working solution contained six parts of oil red $\mathrm{O}$ stock solution $(0.35 \%$ oil red $\mathrm{O}$ in isopropanol) and four parts of $\mathrm{H}_{2} \mathrm{O}$. Cells were washed with distilled water four times and then added with $100 \%$ isopropanol for $10 \mathrm{~min}$ to dissolve the bound staining. Afterward, the absorbance was measured at $500 \mathrm{~nm}$.

\section{Lipolysis measurement}

Lipolysis was evaluated by measuring the amount of glycerol released into the medium [28]. After starvation, differentiated 3T3-L1 adipocytes were treated with PHME $(100,200,300,400$ and $500 \mu \mathrm{g} / \mathrm{mL})$ for 24,48 and $72 \mathrm{~h}$. Then the supernatant of the media was collected for glycerol release measurement using a glycerol assay kit (APPLYGEN Beijing, China).

\section{Protein extraction and Western blotting analysis}

Fully differentiated 3T3-L1 cells treated or untreated with PHME were washed twice with cold PBS buffer and then lysed in a lysis buffer $(150 \mathrm{mM}$ sodium chloride, $1.0 \%$ Triton X-100, $0.5 \%$ sodium deoxycholate, $0.1 \%$ sodium dodecyl sulfate (SDS), $50 \mathrm{mM}$ Tris, $\mathrm{pH}$ 8.0) containing protease and phosphatase inhibitors. Cell lysates were incubated in ice for $30 \mathrm{~min}$, vortexed every $5 \mathrm{~min}$, lysed at $-80^{\circ} \mathrm{C}$ overnight, and then were centrifuged at $12,000 \mathrm{rpm}$ for $20 \mathrm{~min}$ at $4{ }^{\circ} \mathrm{C}$ [28]. The protein concentrations of the supernatant were determined by BCA (bicinchonininc acid) protein assay kit (YEASEN Biotechnology Co., Ltd., Shanghai, China) using BSA (bovine serum albumin) as standard. Proteins $(50 \mu \mathrm{g}$ per lane) were subjected to electrophoresis and separated by $12 \%$ SDS- polyacrylamide gel electrophoresis and transferred to nitrocellulose membranes (ExCell, China). The membranes were blocked in 5\% non-fat drymilk solution at room temperature for $1 \mathrm{~h}$ and incubated with the primary antibodies at $4{ }^{\circ} \mathrm{C}$ for overnight. Then the membranes were washed with TBST (Tris Buffered saline Tween) and then incubated with the secondary antibodies (Gaithersburg, $\mathrm{MD} ; 1: 10000)$ for $1 \mathrm{~h}$ at room temperature. Images were captured using the Odyssey CLx Imaging System (LI-COR, Lincoln, NE) [28].

\section{HPLC analysis \\ Sample preparation}

A stock solution of $2 \mathrm{mg} / \mathrm{mL}$ of PHME was made in 15\% methanol. The solution was ultrasonicated for $5 \mathrm{mins}$ and filtered through a $0.22 \mu \mathrm{m}$ membrane filter before HPLC analysis.

\section{Chromatographic conditions}

HPLC analysis was performed on an Agilent 1100 Series liquid chromatograph system and an ultraviolet detector (Agilent Technologies, Palo Alto, California, USA). Based on previous reports $[2,8,30]$ and preliminary studies, the 
wavelength was selected $260 \mathrm{~nm}$ for uridine, vernine and adenosine, and selected $254 \mathrm{~nm}$ for cordycepin analysis. Separation was conducted on a C18 analytical column $\left(250 \mathrm{~mm} \times 4.6 \mathrm{~mm}\right.$, No.LAAI-KR006, Kromasil) at $30^{\circ} \mathrm{C}$ with a flow rate of $1.0 \mathrm{~mL} / \mathrm{min}$ and injection volume of $10 \mu \mathrm{L}$. In analysis of uridine, vernine and adenosine, the mobile phase consisted of two solvents $(\mathrm{v} / \mathrm{v})$ : $85 \%$ phosphate buffer $\left(\mathrm{Na}_{2} \mathrm{HPO}_{4}-\mathrm{NaH}_{2} \mathrm{PO}_{4}, \mathrm{pH} 6.5\right)$ and $15 \%$ methanol. Meanwhile, the mobile phase was $20 \%$ methanol solution for cordycepin analysis.

\section{Calibration curves}

Four standard samples, uridine $(100 \mu \mathrm{g} / \mathrm{mL})$, vernine $(50 \mu \mathrm{g} / \mathrm{mL})$, adenosine $(80 \mu \mathrm{g} / \mathrm{mL})$ and cordycepin $(50 \mu \mathrm{g} / \mathrm{mL})$ were prepared by dissolving in $15 \%$ methanol, respectively. Then stock solutions were diluted with $15 \%$ methanol to appropriate concentrations for establishing calibration curves. The calibration curves were constructed by plotting the relative peak areas vs. the concentrations of each standard sample.

\section{Precision}

In order to detect the precision and stability of the HPLC system, the precision test, stability test and sample recovery test were executed, respectively. Certain concentrations of standard and sample solutions were tested. Every concentration was tested for at least five times. Variations were expressed by relative standard deviations (R.S.D.).

\section{Statistical analysis}

The data were presented as mean \pm SD and analyzed by one-way ANOVA followed by Duncan's multiple range test using SPSS software (13.0 ed.). $P<0.05$ and $P<0.01$ were considered statistically significant and extremely significant between groups, respectively.

\section{Results}

\section{Effects of PHME on cell viability of 3T3-L1 adipocytes}

The potential cytotoxicity of PHME on fully differentiated 3 T3-L1 adipocytes was evaluated by MTT assay. As results shown in Fig. 1, 200 and $300 \mu \mathrm{g} / \mathrm{mL}$ PHME didn't affect the viability of 3T3-L1 adipocytes $(P>0.05)$. Treatment with 400 and $500 \mu \mathrm{g} / \mathrm{mL}$ PHME for $24 \mathrm{~h}$ increased the cell viability compared with control cells by 6.5 and $5.3 \%$, respectively $(P<0.01)$. Our data showed that PHME had no cytotoxic effect against fully differentiated 3T3-L1 adipocytes. Therefore, PHME at concentrations less than $500 \mu \mathrm{g} / \mathrm{mL}$ were used in the following study.

\section{PHME stimulated basal lipolysis in 3T3-L1 adipocytes}

Triglyceride hydrolysis releases glycerol and free fatty acids from adipocytes. Contents of glycerol released into the medium and intracellular triglyceride were assayed as indices of lipolysis. Fully differentiated 3T3-L1 adipocytes were incubated with various concentrations of PHME for 24, 48 and $72 \mathrm{~h}$. As results shown in Fig. 2a, 200, 300, 400 and $500 \mu \mathrm{g} / \mathrm{mL}$ PHME significantly increased glycerol contents in the medium $(P<0.05$ or $P<$ $0.01)$, whereas $100 \mu \mathrm{g} / \mathrm{mL}$ PHME hardly exhibited any effect on glycerol release $(P>0.05)$. Oil red $\mathrm{O}$ staining was performed to confirm lipolytic effect of PHME, and results (Fig. 2b) showed that all concentrations of PHME significantly decreased the content of intracellular triglyceride after $72 \mathrm{~h}$ of treatment $(P<0.01)$. These results revealed PHME significantly stimulated basal lipolysis in mature 3T3-L1 adipocytes.

\section{Effects of PHME on the main lipases and kinases involved in lipolysis regulation}

ATGL, HSL and perilipin have been reported as the major regulators for lipolysis control. To investigate the underlying molecular mechanisms by which PHME stimulates adipocyte lipolysis, we performed western blotting analysis to examine the involvement of these regulators. As results shown in Fig. 3a, PHME treatment (200 and $400 \mu \mathrm{g} / \mathrm{mL}$ ) did not modify protein expression of ATGL levels, but it increased PKA-dependent phosphorylation of HSL at Ser563 and Ser660. Moreover, the protein expression of perilipin was markedly downregulated by PHME treatment (Fig. 3a).

AMP-activated protein kinase (AMPK) and mitogen-activated protein kinases (MAPKs) signaling pathways were involved in the regulation of lipolysis. In this study, we tested the phosphorylation of AMPK and three MAPKs. As shown in Fig. 3a and b, PHME treatment did not affect phosphorylation of AMPK and AMPK-mediated phosphorylation of HSL at Ser565. After 24 h treatment, PHME upregulated the phosphorylation of ERK, but it did not modify the phosphorylation of JNK and p38 (Fig. 3b). These results suggested that PKA and ERK signaling pathways were probably involved in PHME-promoted adipocyte lipolysis.

\section{PHME increased HSL phosphorylation through activation of PKA pathway}

To further investigate whether PKA pathway was involved in mediating PHME-stimulated lipolysis, fully differentiated 3T3-L1 adipocytes were treated with PHME for $12 \mathrm{~h}$ with or without H89 (PKA inhibitor), and then glycerol release were measured. As glycerol contents showed in Fig. 4a, $200 \mu \mathrm{g} / \mathrm{mL}$ PHME significantly promoted content of glycerol released into the medium $(P<0.01$ vs. control group), and this effect was abolished by pretreatment with H89 $(P<0.01$ vs. PHME-treated alone). Furthermore, the blocking of PKA also reversed PHME-induced upregulated phosphorylation of HSL at residues of Ser563 and Ser660 (Fig. 4b). These results indicated that PHME upregulated HSL phosphorylation through activation of PKA pathway, supporting the key role of the PKA pathway in lipolytic action of PHME. 


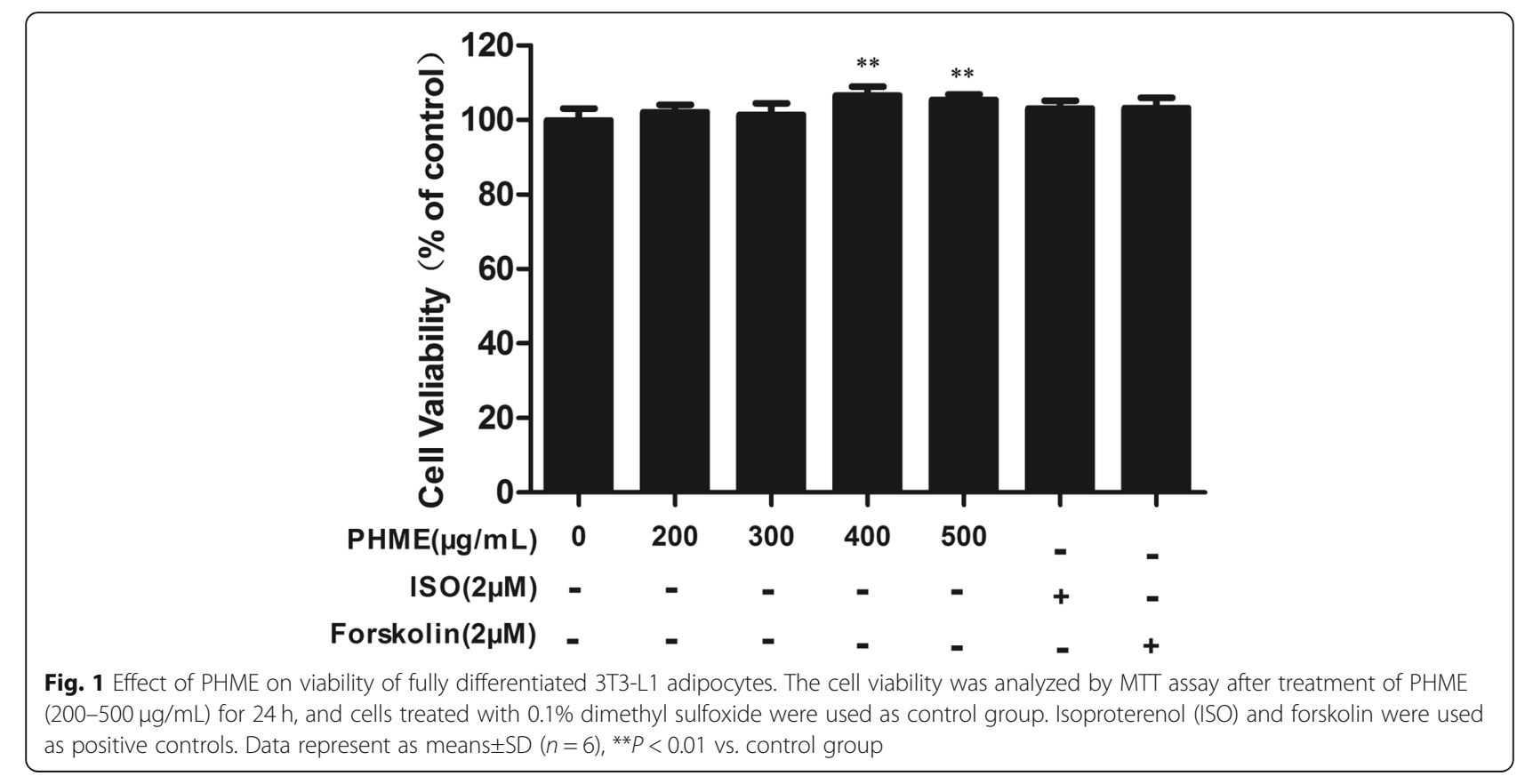

PHME downregulated the protein expression of perilipin through ERK activation

To investigate the possible role of the ERK pathway in PHME-stimulated lipolysis, an ERK inhibitor (PD98059) was employed to block activation of the ERK pathway. First, the effect of PD98059 on PHME-stimulated adipocyte lipolysis was evaluated. As results showed in Fig. 5a, after treatment for $12 \mathrm{~h}$, PHME significantly promote glycerol release in the culture $(P<0.05$ vs. control group). Pretreatment with PD98059 attenuated PHME-caused glycerol release $(P<0.01$ vs. PHME-treated group). Furthermore, PHME treatment decreased the protein expression of
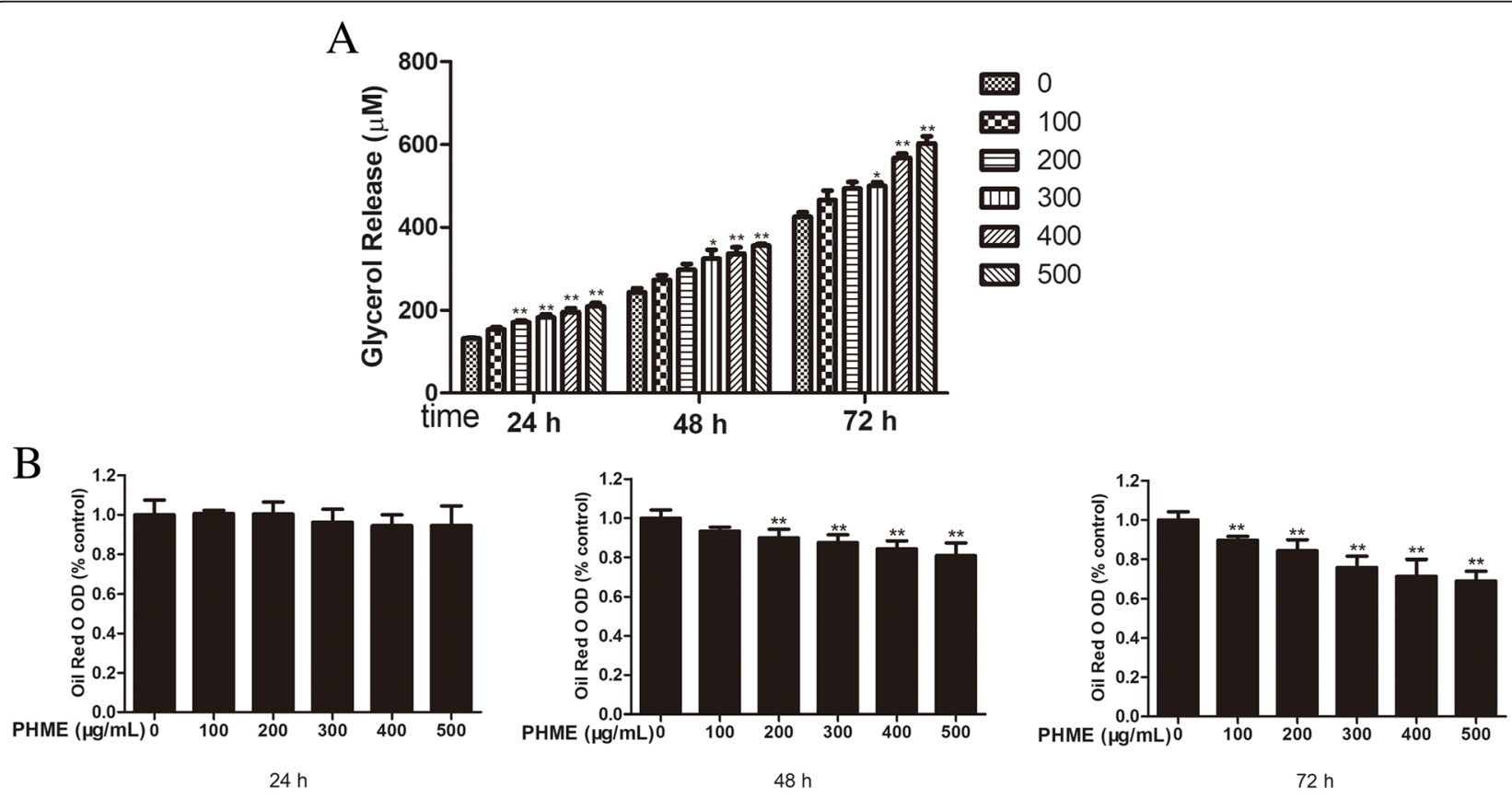

Fig. 2 Effect of PHME on adipocyte lipolysis. a Fully differentiated adipocytes treated with various concentrations of PHME (100, 200, 300, 400 and $500 \mu \mathrm{g} / \mathrm{mL}$ ) for 24, 48 and $72 \mathrm{~h}$, respectively. Then, glycerol contents in media were measured by glycerol assay kit. b Adipocytes treated with PHME for 24, 48 and $72 \mathrm{~h}$ were stained with oil red $\mathrm{O}$ and quantified intracellular lipid by measuring the absorbance at 500 nm. Values are expressed as mean \pm SD $(n=4)$, ${ }^{*} P<0.05$ and ${ }^{*} P<0.01$ vs. control group, respectively 

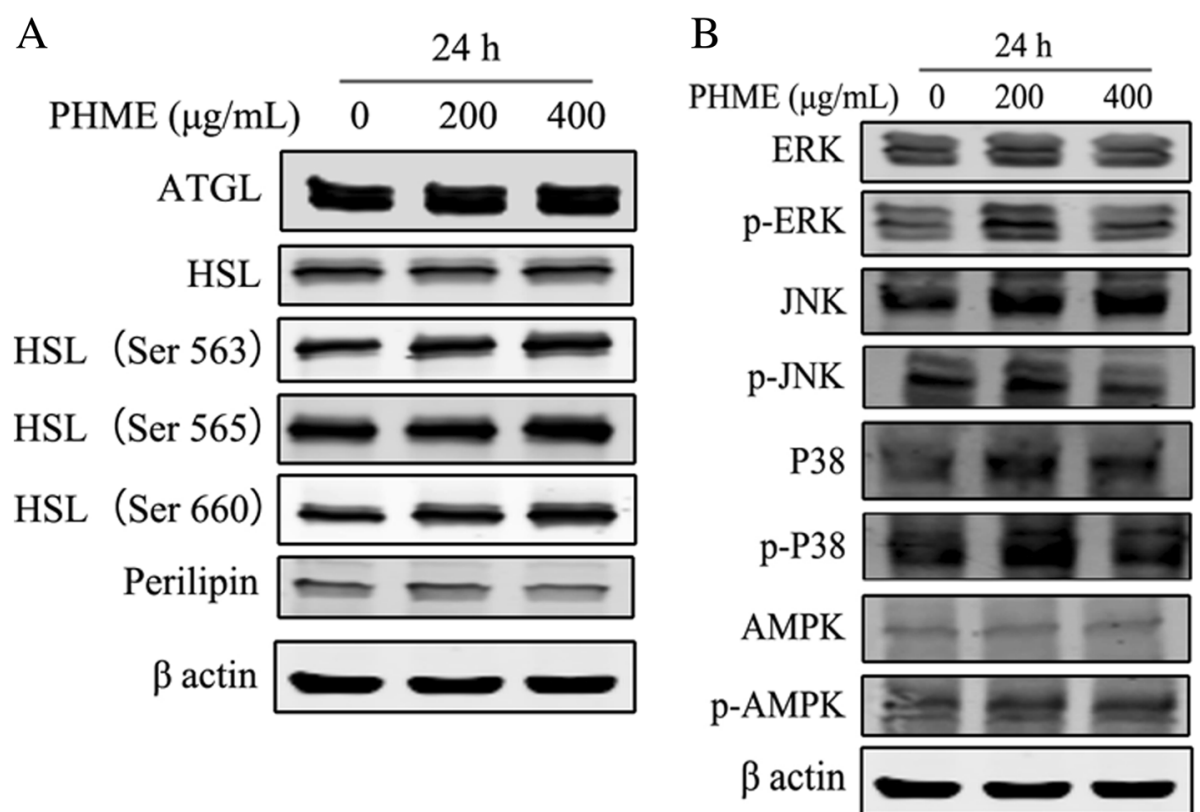

Fig. 3 Effect of PHME on the expression of ATGL, perilipin, HSL and phosphorylated HSL at Ser563, Ser565 and Ser660 (a), and on the expression

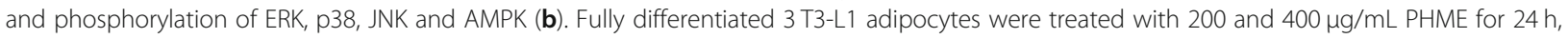
then expression of ATGL and perilipin, as well as basal and phosphorylated levels of HSL, AMPK and MAPKs were measured using western blotting analysis

perilipin, and this phenomenon was also abolished by PD98059 treatment (Fig. 5b). The results suggested that PHME stimulated lipolysis by decreasing perilipin via activation of ERK pathway.

\section{HPLC analysis of PHME}

HPLC analysis of PHME revealed four peaks matching commercial standards: uridine, vernine, adenosine, and cordycepin, with retention times of approximately 4.14, 5.56, 11.36 and $4.41 \mathrm{~min}$, respectively (Additional file 1: Figure S1).
Quantification of nucleosides was analyzed using standard curves. According to the UV spectra of analytes, $260 \mathrm{~nm}$ was used as detection wavelength for uridine, vernine, and adenosine, as well as $254 \mathrm{~nm}$ was used as detection wavelength for cordycepin. As shown in Additional file 2: Table S1, a satisfactory linear relationship was obtained between the concentrations of the analyte and corresponding peak area. The precision results demonstrated that the developed method is precise and sensitive for the quantitative determination of uridine, vernine, adenosine, and cordycepin in PHME. Moreover, the calibration curves of four analytes were calculated by plotting the
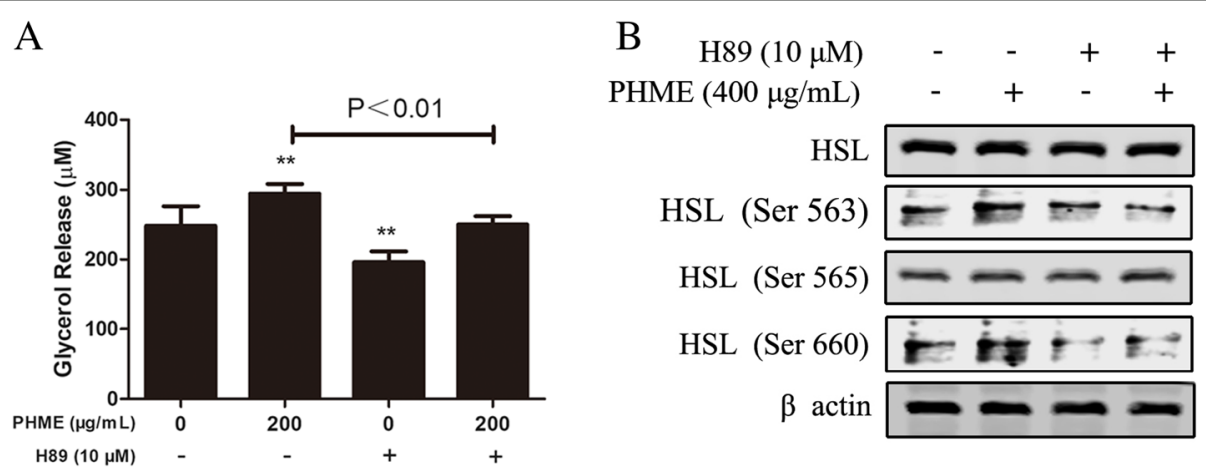

Fig. 4 Effects of a PKA inhibitor (H89) on PHME-stimulated lipolysis. a Fully differentiated 3T3-L1 adipocytes were treated with $200 \mu \mathrm{gg} / \mathrm{mL}$ PHME alone or in the presence of $10 \mu \mathrm{M} \mathrm{H} 89$ for $12 \mathrm{~h}$, then glycerol released into the medium was quantified. $\mathbf{b}$ Fully differentiated 3T3-L1 adipocytes were treated with $400 \mu \mathrm{g} / \mathrm{mL}$ PHME alone or in the presence of $10 \mu \mathrm{M} \mathrm{H} 89$ for $12 \mathrm{~h}$, then cells were lysed, and then basal and phosphorylated levels of HSL were measured using western blotting analysis. Values are expressed as mean $\pm \operatorname{SD}(n=4)$, ** $P<0.01$ vs. control group 
A

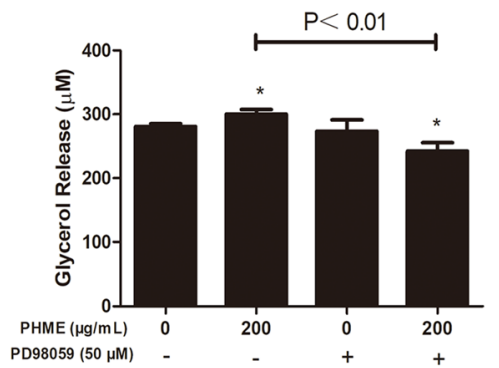

$\begin{array}{lllll}\text { B } \\ \text { PD98059 }(50 \mu \mathrm{M}) & - & - & + & + \\ \text { PHME }(200 \mu \mathrm{g} / \mathrm{mL}) & - & + & - & +\end{array}$

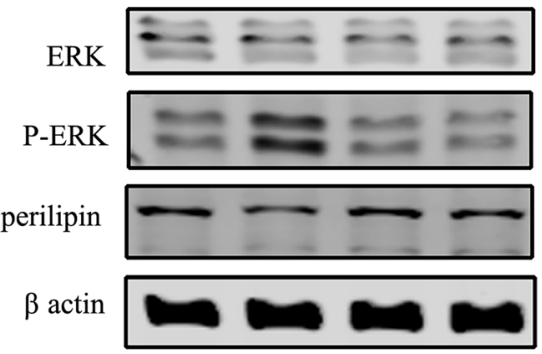

Fig. 5 Effects of an ERK inhibitor (PD98059) on PHME-stimulated lipolysis. a Fully differentiated 3T3-L1 adipocytes were treated with $200 \mu \mathrm{g} / \mathrm{mL}$ PHME alone or in the presence of $50 \mu \mathrm{M}$ PD98059 for $12 \mathrm{~h}$, then contents of glycerol released into the medium were quantified. $\mathbf{b}$ Fully differentiated 3T3-L1 adipocytes were treated with $200 \mathrm{\mu g} / \mathrm{mL}$ PHME alone or in the presence of $50 \mathrm{\mu M}$ PD98059 for $20 \mathrm{~h}$, then cells were lysed, and then ERK phosphorylation and expression of perilipin were measured using western blotting analysis. Values are expressed as mean $\pm \mathrm{SD}(n=4)$, * $P<0.05$ vs. control group

peak areas (y) vs. the corresponding concentrations $(\mathrm{x}, \mu \mathrm{g} / \mathrm{mL})$ by using the standard solutions (Additional file 2: Table S1). The amount of four analytes were calculated by calibration curves listed in Additional file 2: Table S1, respectively. The contents of uridine, vernine, adenosine and cordycepin in PHME were $0.94 \pm 0.002,0.44 \pm$ $0.001,0.33 \pm 0.001$ and $0.53 \% \pm 0.001(n=5)$, respectively.

\section{Discussion}

In the current study, we found PHME exhibited lipolytic activity in 3T3-L1 adipocytes. This extract significantly stimulated adipocyte lipolysis and reduced intracellular triglyceride accumulation through activation of HSL and downregulation of perilipin (Fig. 6). PKA and ERK signal pathways were involved in mediating the lipolytic effect of PHME.

In this study, we found that PHME increased phosphorylation of HSL at Ser563 and Ser660. It is well-known that phosphorylation of HSL at Ser563, Ser659 and Ser660 by PKA activates adipocyte lipolysis [22]. PKA phosphorylates HSL resulting in increased hydrolytic activity, translocation of HSL from cytosol to the lipid droplet surface, and hydrolyzed DAG [31]. Our results suggested that PHME enhanced glycerol release probably through activation of PKA pathway. Then, an inhibitor of PKA, H89, was used to confirm this hypothesis and found H89 blunted the PHME caused phosphorylation of HSL at Ser563 and Ser660 (major PKA-targets), and the subsequent increase in glycerol release. These findings revealed that PHME -stimulated lipolysis was dependent on PKA activation.

Moreover, we observed that PHME treatment caused a significant decrease in protein expression of perilipin A. Perilipin A plays an essential role in the control of lipolysis [25]. In basal condition, perilipin A acts as a barrier to lipases, thereby maintaining a low rate of basal lipolysis [22]. Thus, a decrease in perilipin A expression allows access of the lipases to the lipid droplet and enhanced lipolysis [25]. The ERK signaling pathway is involved in regulating perilipin protein expression and perilipin phosphorylation. ERK activation is able to regulate adipocyte lipolysis by phosphorylating HSL at Ser600 and therefore increasing the activity of HSL [32]. In addition, ERK activation is reported as an early signal for the reduction in perilipin protein expression and subsequent induction of lipolysis [24]. Previous studies revealed that TNF- $\alpha$ stimulated lipolysis in 3T3-L1 and human adipocytes are dependent on down-regulation of perilipin expression via activation of ERK pathway $[26,33]$.

In the present study, incubation with PHME resulted in a significant increase in lipolysis, which were paralleled by a decrease in the protein expression of perilipin A and an increase in level of phosphorylated ERK. It is known that decreased protein expression may impair the barrier function of perilipin A and thus lead to the increased lipolysis. Thus, we speculate that downregulating of perilipin A expression caused by PHME is one of its lipolytic mechanisms, and ERK pathway probably be involved in regulation of perilipin A expression. To test this possibility, we used PD98059 (an inhibitor of ERK) to block the ERK activity during 3T3-L1 adipocyte lipolysis and found that both PHME-caused lipolysis and downregulation of perilipin A expression were inhibited in the presence of PD98059. Therefore, these results indicated that PHME-caused lipolysis is dependent on down-regulation of perilipin expression via ERK pathway.

AMPK pathway also involves in the regulation of lipolysis. Indeed, translocation of HSL to the lipid droplets inhibited by AMPK through phosphorylation at Ser565 is well established [25]. Our current data showed that phosphorylation of HSL at Ser565 and phosphorylated AMPK level weren't affected by PHME treatment. These results indicated that AMPK pathway wasn't involved in mediating the lipolytic effect of PHME. 


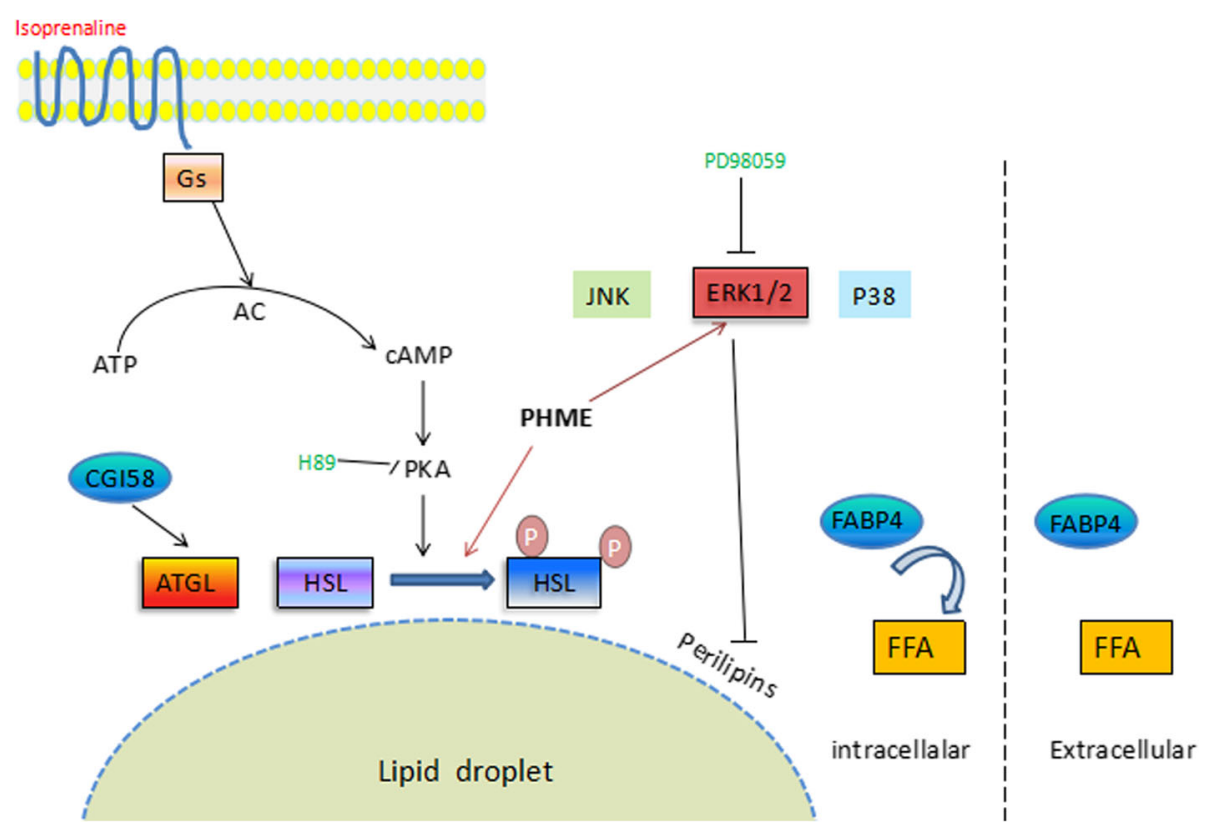

Fig. 6 Mechanisms for the lipolytic effect of PHME in 3T3-L1 adipocytes. PHME stimulates lipolysis via activating HSL and downregulating perilipin through PKA and ERK signaling pathways

Nucleosides, one of the major ingredients in Cordyceps, are believed to be the active components in Cordyceps and used as marker for quality control of Cordyceps [2, 3]. Such as adenosine is a marker for quality control of $C$. sinensis [2]. As the important fungal species isolated from natural C. sinensis, Paecilomyces hepiali also contain bioactive nucleosides which used as marker for quality control. It is known the amount and sources of nucleosides in natural Cordyceps are different from that of cultured one [2]. Moreover, the content and sources of nucleosides in cultured mycelia are affected by submerged culture conditions and nutritional requirements. Therefore, main constituents of nucleosides were analyzed in the present study. Results of HPLC showed there were four nucleosides including adenosine, cordycepin, uridine and vernine in PHME. In order to clarify the active compound, we further evaluated effect of these four nucleosides on adipocyte lipolysis, and found only cordycepin exhibited significant lipolytic action (Additional file 3: Figure S2). Cordycepin were reported to possess anti-cancer, anti-oxidant and immunomodulating activities [34]. Moreover, cordycepin suppressed differentiation of 3T3-L1 pre-adipocytes and pre-adipocytes in primary culture, and this anti-adipogenic effect occur through its intervention in the mTORC1-C/EBP $\beta-P P A R \gamma$ pathway [35]. Therefore, it is suggested that cordycepin might be one of the main active constituents in PHME that could enhance lipolysis via the PKA and ERK signaling pathways. However, further studies would be crucial to investigate mechanism of lipolytic effect of cordycepin.

\section{Conclusions}

In summary, the present data demonstrate that methanol extract from mycelia of Paecilomyces hepiali induced HSL phosphorylation and decreased perilipin level, therefore increased lipolysis in fully differentiated 3T3-L1 adipocytes. PHME-induced lipolysis was mediated via at least two different pathways. One involved PKA activation, leading to phosphorylation of HSL at Ser 563 and 660 to hydrolyze triacylglycerols in adipocytes. In the other pathway, PHME induced the phosphorylation of ERK, thereby decreasing the levels of perilipin to promote lipolysis. $P$. hepiali has been widely used as a medicine or a dietary supplement. Our results provided a potential mechanism by which $P$. hepiali could improve lipid disorders.

\section{Additional files}

Additional file 1: Figure S1. HPLC analysis of PHME. (A) HPLC chromatogram of PHME at $260 \mathrm{~nm}$. (B) HPLC chromatogram of PHME at $254 \mathrm{~nm}$. Uridine (1), vernine (2), adeosine (3), and cordycepin (4) were determined, respectively. (TIF $459 \mathrm{~kb}$ )

Additional file 2: Table S1. Linear regression data and precision of four nucleosides (DOCX $17 \mathrm{~kb}$ )

Additional file 3: Figure S2. Effects of adenosine, vernine, uridine, mannitol and cordycepin on lipolysis in 3T3-L1 adipocytes. Mature 3T3-L1 adipocytes were treated with 10-100 $\mu \mathrm{mol} / \mathrm{L}$ Adenosine $(A)$, vernine $(B)$, uridine (C), mannitol (D) for $24 \mathrm{~h}$, and 10-100 $\mu \mathrm{mol} / \mathrm{L}$ cordycepin (E) for 24, 48, and $72 \mathrm{~h}$, then the amounts of glycerol released into the culture medium were measured. Values are expressed as mean $\pm \mathrm{SD}(n=4)$, ${ }^{* *} P<0.01$ vs. control group. (TIF 419 kb) 


\section{Abbreviations}

AMPK: AMP-activated protein kinase; ATGL: Adipose triglyceride lipase; ERK: Extracellular signal-regulated kinase; FA: Fatty acids; HPLC: High performance liquid chromatography; HSL: Hormone-sensitive lipase; MAPK: Mitogen-activated protein kinase; PHME: Methanol extract of Paecilomyces hepiali mycelia; PKA: Protein kinase A; TG: Triglyceride; WAT: White adipose tissue

\section{Acknowledgements}

The authors are particularly grateful to Prof. Yu-quan Xu and Jing-ya Li for their help.

\section{Funding}

This study was supported by grants from the Advanced Industrial Technology Research Institute of Shanghai Jiaotong University (Shanghai, China).

\section{Availability of data and materials}

The data was included in figures of the manuscript, and the raw data for this study are available upon reasonable request to the corresponding author.

\section{Authors' contributions}

WZ and MG designed the study, analyzed the data and wrote the manuscript. MG carried out most experiments. RG and HXL assisted in all experiments. All authors read and approved the final manuscript.

\section{Ethics approval and consent to participate}

Not applicable.

\section{Consent for publication}

Not applicable.

\section{Competing interests}

All authors declare that they have no competing interests.

\section{Publisher's Note}

Springer Nature remains neutral with regard to jurisdictional claims in published maps and institutional affiliations.

Received: 10 May 2018 Accepted: 27 November 2018 Published online: 07 December 2018

\section{References}

1. Huang LF, Liang YZ, Guo FQ, Zhou ZF, Cheng BM. Simultaneous separation and determination of active components in Cordyceps sinensis and Cordyceps militarris by LC/ESI-MS. J Pharm Biomed Anal. 2003:33:1155-62

2. Li SP, Yang FQ, Tsim KW. Quality control of Cordyceps sinensis, a valued traditional Chinese medicine. J Pharm Biomed Anal. 2006:41:1571-84.

3. Lin B, Li S. Cordyceps as an herbal drug. In: Benzie IFF, Wachtel-Galor S, editors. Herbal medicine: biomolecular and clinical aspects. 2nd ed. Boca Raton (FL): CRC Press/Taylor \& Francis; 2011. Chapter 5. https://www.ncbi.nlm.nih.gov/books/ NBK92758/.

4. Bok JW, Lermer L, Chilton J, Klingeman HG, Towers GH. Antitumor sterols from the mycelia of Cordyceps sinensis. Phytochemistry. 1999;51:891-8

5. Kuo YC, Tsai WJ, Wang JY, Chang SC, Lin CY, Shiao MS. Regulation of bronchoalveolar lavage fluids cell function by the immunomodulatory agents from Cordyceps sinensis. Life Sci. 2001;68:1067-82.

6. Lo HC, Hsieh C, Lin FY, Hsu TH. A systematic review of the mysterious caterpillar fungus Ophiocordyceps sinensis in dong-ChongXiaCao (冬蟲夏草 Dōng Chóng Xià Căo) and related bioactive ingredients. J Tradit Complement Med. 2013;3:16-32.

7. Zhang G, Huang Y, Bian Y, Wong JH, Ng TB, Wang H. Hypoglycemic activity of the fungi Cordyceps militaris, Cordyceps sinensis, Tricholoma mongolicum, and Omphalia lapidescens in streptozotocin-induced diabetic rats. Appl Microbiol Biotechnol. 2006;72:1152-6.

8. Zhao J, Xie J, Wang LY, Li SP. Advanced development in chemical analysis of Cordyceps. J Pharm Biomed Anal. 2014;87:271-89.

9. Zhu JS, Gao L, Li XH, Yao YS, Zhao JQ, Zhou YJ, et al. Maturational alteration of oppositely orientated rDNA and differential proliferation of GC- and ATbiased genotypes of Ophiocordyceps sinensis and Paecilomyces hepiali in natural Cordyceps sinensis. Am J Biomed Sci. 2010;2:217-38.
10. Wu Z, Zhang M, Xie M, Dai Z, Wang X, Hu B, et al. Extraction, characterization and antioxidant activity of mycelial polysaccharides from Paecilomyces hepiali HN1. Carbohydr Polym. 2016;137:541-8.

11. Wang J, Li LZ, Liu YG, Teng LR, Lu JH, Xie J, et al. Investigations on the antifatigue and antihypoxic effects of Paecilomyces hepiali extract. Mol Med Rep. 2016:13:1861-8.

12. Wang C, Wang J, Jia D, Li L, Jia B, Fan S, et al. Anti-nociceptive effects of Paecilomyces hepiali via multiple pathways in mouse models. Genet Mol Res. 2016;15. https://doi.org/10.4238/gmr.15038996.

13. Wu Z, Lu J, Wang X, Hu B, Ye H, Fan J, et al. Optimization for production of exopolysaccharides with antitumor activity in vitro from Paecilomyces hepiali. Carbohydr Polym. 2014;99:226-34.

14. Park SY, Jung SJ, Ha KC, Sin HS, Jang SH, Chae HJ, et al. Anti-inflammatory effects of Cordyceps mycelium (Paecilomyces hepiali, CBG-CS-2) in Raw264.7 murine macrophages. Orient Pharm Exp Med. 2015:15:7-12.

15. Wang J, Teng L, Liu Y, Hu W, Chen W, Hu X, et al. Studies on the antidiabetic and antinephritic activities of Paecilomyces hepiali water extract in diet-streptozotocin-induced diabetic Sprague dawley rats. J Diabetes Res. 2016;2016:4368380. https://doi.org/10.1155/2016/ 4368380

16. Holm C. Molecular mechanisms regulating hormone-sensitive lipase and lipolysis. Biochem Soc Trans. 2003;31:1120-4.

17. El Husseny MW, Mamdouh M, Shaban S, Ibrahim Abushouk A, Zaki MM, Ahmed OM, et al. Adipokines: potential therapeutic targets for vascular dysfunction in type II diabetes mellitus and obesity. J Diabetes Res. 2017; 2017:8095926.

18. Kata FS, Athbi AM, Manwar EQ, Al-Ashoor A, Abdel-Daim MM, Aleya L. Therapeutic effect of the alkaloid extract of the cyanobacterium Spirulina platensis on the lipid profile of hypercholesterolemic male rabbits. Environ Sci Pollut Res Int. 2018. https://doi.org/10.1007/s11356018-2170-4

19. Ahmadian M, Duncan RE, Sul HS. The skinny on fat: lipolysis and fatty acid utilization in adipocytes. Trends Endocrinol Metab. 2009;20:424-8.

20. Jaworski K, Sarkadi-Nagy E, Duncan RE, Ahmadian M, Sul HS. Regulation of triglyceride metabolism. IV. Hormonal regulation of lipolysis in adipose tissue. Am J Physiol Gastrointest Liver Physiol. 2007;293:G1-4

21. Brasaemle DL. Lipolysis control: the plot thickens. Cell Metab. 2010;11:173-4.

22. Frühbeck G, Méndez-Giménez L, Fernández-Formoso JA, Fernández $S$, Rodríguez A. Regulation of adipocyte lipolysis. Nutr Res Rev. 2014;27:63-93.

23. Lafontan M, Langin D. Lipolysis and lipid mobilization in human adipose tissue. Prog Lipid Res. 2009;48:275-97.

24. Zechner R, Zimmermann R, Eichmann TO, Kohlwein SD, Haemmerle G, Lass A, et al. Fat signals-lipases and lipolysis in lipid metabolism and signaling. Cell Metab. 2012;15:279-91.

25. Arner $\mathrm{P}$, Langin D. Lipolysis in lipid turnover, cancer cachexia, and obesityinduced insulin resistance. Trends Endocrinol Metab. 2014:25:255-62.

26. Souza SC, Palmer HJ, Kang YH, Yamamoto MT, Muliro KV, Paulson KE, et al. TNF-alpha induction of lipolysis is mediated through activation of the extracellular signal related kinase pathway in 3T3-L1 adipocytes. J Cel Biochem. 2003:89:1077-86.

27. Malucká LU, Harvanova J, Uhrinová A, Salayová A, Pavlík M, Rajtar M. Antioxidant activity and infrared spectroscopy analysis of alcoholic extracts obtained from Paecilomyces hepiali (ascomycetes). Int J Med Mushrooms. 2018;20:595-605.

28. Wang $\mathrm{Q}$, Wang ST, Yang X, You PP, Zhang W. Myricetin suppresses differentiation of 3T3-L1 preadipocytes and enhances lipolysis in adipocytes. Nutr Res. 2015:35:317-27.

29. Han JH, Kim IS, Jung SH, Lee SG, Son HY, Myung CS. The effects of propionate and valerate on insulin responsiveness for glucose uptake in 3T3-L1 adipocytes and C2C12 myotubes via G protein-coupled receptor 41. PLoS One. 2014;9:e95268. https://doi.org/10.1371/journal. pone.0095268.

30. Cheng W, Zhang X, Song Q, Lu W, Wu T, Zhang Q, Li C. Determination and comparative analysis of 13 nucleosides and nucleobases in natural fruiting body of Ophiocordyceps sinensis and its substitutes. Mycology. 2017;8:318-26.

31. Ahmadian M, Wang Y, Sul HS. Lipolysis in adipocytes. Int J Biochem Cell Biol. 2010;42:555-9.

32. Greenberg AS, Shen WJ, Muliro K, Patel S, Souza SC, Roth RA, et al. Stimulation of lipolysis and hormone-sensitive lipase via the extracellular signal-regulated kinase pathway. J Biol Chem. 2001;276:45456-61. 
33. Rydén M, Arvidsson E, Blomqvist L, Perbeck L, Dicker A, Arner P. Targets for TNF-a-induced lipolysis in human adipocytes. Biochem Biophys Res Commun. 2004;318:168-75

34. Tuli HS, Sandhu SS, Sharma AK. Pharmacological and therapeutic potential of Cordyceps with special reference to cordycepin. 3 Biotech. 2014;4:1-12.

35. Takahashi S, Tamai M, Nakajima S, Kato H, Johno H, Nakamura T, et al. Blockade of adipocyte differentiation by cordycepin. Br J Pharmacol. 2012; 167:561-75.

Ready to submit your research? Choose BMC and benefit from:

- fast, convenient online submission

- thorough peer review by experienced researchers in your field

- rapid publication on acceptance

- support for research data, including large and complex data types

- gold Open Access which fosters wider collaboration and increased citations

- maximum visibility for your research: over $100 \mathrm{M}$ website views per year

At $\mathrm{BMC}$, research is always in progress.

Learn more biomedcentral.com/submissions 